\title{
CARACTERIZAÇÃO DOS PARTOS E NASCIDOS VIVOS DE MÃES RESIDENTES EM UM MUNICÍPIO DE GOIÁS, BRASIL
}

\author{
CHARACTERIZATION OF BIRTHS AND LIVE BIRTHS OF MOTHERS LIVING IN A MUNICI- \\ PALITY OF GOIÁS, BRAZIL
}

\section{Marília Carvalho Moreira da Silva ${ }^{\mathrm{a}^{*}}$, Gabriela Camargo Tobias ${ }^{\mathrm{b}^{* *}}$, Cristiane Chagas Teixeira ${ }^{\mathrm{c}^{* *}}$}

mariliac.m.dasilva@hotmail.com ${ }^{\mathrm{a}}$, gabicamargo22@gmail.com ${ }^{\mathrm{b}}$,cc-teixeira@hotmail.com Universidade Estadual de Goiás*, Universidade Federal de Goiás*,

Data de Submissão:03/10/2018

Data de Aceite: 02/10/2019

\section{RESUMO}

Objetivo: caracterizar partos e nascidos vivos de mães residentes em um município de Goiás, Brasil. Método: estudo de corte transversal, quantitativo, com dados de 186 nascimentos ocorridos no período de $1^{\circ}$ de janeiro a 31 de dezembro de 2016. A amostra foi composta pelos nascidos vivos, filhos de mães residentes no município de Caiapônia, Goiás, Brasil. A coleta de dados foi feita no site do Departamento de Informática do SUS (DATASUS). Resultados: predominância dos partos hospitalares (99,46\%) e sexo masculino (51,61\%), com frequência reduzida de baixo peso ao nascer (6,46\%). Com relação às mães, observou-se 17,64\% adolescentes e $12,37 \%$ com menos de oito anos de estudo. Em relação aos nascidos vivos, 93,55\% foram a termo, $86,02 \%$ de partos cesáreos e $68,81 \%$ realizaram mais de 7 consultas de pré-natal. Conclusão: houve melhoria da qualidade das informações do Sistema de Informações sobre Nascidos Vivos, pela redução do percentual de preenchimento da categoria "ignorado", podendo ser considerado como poderoso instrumento para o monitoramento e avaliação da realidade local, servindo de ferramenta para o planejamento de ações no âmbito da saúde da mulher e da criança.

Palavras-chave: Sistemas de informação; saúde materno-infantil; nascimento vivo; atenção primária à saúde; enfermagem.

\section{ABSTRACT}

Objective: to characterize births and live births of mothers living in a municipality of Goiás, Brazil. Method: a cross-sectional, quantitative study with data from 186 births from January 1 to December 31, 2016. The sample was composed of live births, children of mothers living in the municipality of Caiapônia, Goiás, Brazil. Data collection was done on the website of the Department of Informatics of SUS (DATASUS). Results: predominance of hospital deliveries (99.46\%) and male (51.61\%), with low frequency of low birth weight (6.46\%). Regarding the mothers, we observed $17.64 \%$ adolescents and $12.37 \%$ with less than eight years of study. In relation to live births, $93.55 \%$ were at term, $86.02 \%$ of cesarean deliveries and $68.81 \%$ performed more than 7 prenatal consultations. Conclusion: there was an improvement in the information quality of the Live Birth Information System, by reducing the percentage of completion of the "ignored" category, and it can be considered as a powerful instrument for monitoring and evaluating the local reality, serving as a tool for planning the health of women and children.

Keywords: Information systems; maternal and child health; live birth; primary health care; nursing. 


\section{Introdução}

A informação é um instrumento essencial para a tomada de decisões sendo uma ferramenta imprescindível à Vigilância Epidemiológica ${ }^{1}$, por subsidiar o processo "informação-decisão-ação". Ela orienta a implantação, acompanhamento e avaliação dos modelos de atenção à saúde e das ações de prevenção e controle de doenças, se tornando a base para a gestão dos serviços. Para que sejam de qualidade, devem ter sensibilidade para captar o mais precocemente possível as alterações ocorridas, devem ser atuais e mostrar organização e cobertura das atividades, para que todo Sistema de Vigilância Epidemiológica apresente um bom desempenho ${ }^{1-3}$.

O Sistema de Informação em Saúde fornece informações acerca da saúde da população e são constituídos por vários subsistemas que têm como objetivo facilitar a formulação e avaliação das políticas, planos e programas de saúde, subsidiando o processo de tomada de decisões, tomando como referencial microrregiões homogêneas e considerando, necessariamente, às condições de vida da população na determinação do processo saúde-doença. Devem contar com os requisitos técnicos e profissionais necessários ao planejamento, coordenação e supervisão das atividades relativas à coleta, ao registro, ao processamento, à análise, à apresentação e à difusão de dados e geração de informações ${ }^{4-5}$.

O Ministério da Saúde gerencia vários sistemas, por exemplo, o Sistema de Informação da Atenção Básica (SIAB e-SUS), que fornece informações relacionadas ao PACS/PSF/ESF; o Sistema de Informações sobre Mortalidade (SIM), que presta informações sobre as mortes ocorridas no país; o Sistema de Informações sobre Nascidos Vivos (SINASC), que apresenta informações sobre os nascimentos registrados ${ }^{4}$.

Tendo em vista as limitações das técnicas demográficas baseadas em pesquisas por amostragem para estimação da mortalidade, estudos internacionais apontam esforços para a melhoria do sistemas de informações ${ }^{1-2}$. No caso do Brasil, no que se refere às informações vitais, dispõe de dois sistemas: o Sistema de Informação sobre Mortalidade e o Sistema de Informação sobre Nascidos Vivos ${ }^{6}$.
O SINASC fornece informações sobre as características dos nascidos vivos, fundamental para o estabelecimento de indicadores de saúde específicos ${ }^{4}$. Foi implantado a partir de 1990, pelo Ministério da Saúde, que tem como instrumento padronizado de coleta de dados a Declaração de Nascido Vivo, cuja emissão é de competência exclusiva do Ministério da Saúde e que vem apresentando nos últimos anos, um volume maior de registros do que o publicado em anuários do Instituto Brasileiro de Geografia e Estatística (IBGE), com base nos dados de Cartórios de Registro Civil ${ }^{3}$.

Estudo avaliou os atributos e utilidade do SINASC, Brasil, 2006-2010, e apontou que das 23 variáveis avaliadas, 21 apresentaram completude superior a $90,0 \%$; o percentual de completude de variáveis preenchidas em partos hospitalares foi 97,9\%; não houve diferença entre as proporções de nascimentos segundo macrorregiões e sexo, na comparação com os dados do Censo Demográfico 2010. Foram recebidos em tempo oportuno $82,6 \%$ do volume de dados, em 2010; a razão entre nascidos vivos informados e estimados foi de 89,4\% em 2006 e de 97,4\% em 2010; dados do SINASC eram utilizados em 22 indicadores de monitoramento ${ }^{7}$. Outro estudo brasileiro indicou cobertura precária do SINASC, sendo inferior a $60 \%$ em três dos cinco municípios estudados. Quase um quarto dos nascidos vivos subenumerados não teve Declaração de Nascido Vivo emitida. As principais fontes de informação foram os cartórios, hospitais e Unidades Básicas de Saúde, correspondendo juntas ao total de 60\% dos nascidos vivos localizados pela busca ativa ${ }^{5}$.

Nos últimos anos, o Governo Federal tem realizado ações e campanhas para reduzir o subregistro de nascimentos no país e possibilitar o acesso do brasileiro à documentação básica. Uma ação fundamental tem sido a implantação do uso obrigatório em todo o território nacional da Declaração de Nascido Vivo, documento padrão do Ministério da Saúde, hábil para a lavratura da Certidão de Nascimento pelos Cartórios de Registro Civil e, portanto, para a garantia dos direitos de cidadania ${ }^{8}$.

A emissão de Declaração de Nascido Vivo é obrigatória para todo nascido vivo, realizada no município de ocorrência do nascimento, 
independente da duração da gestação, peso e estatura do recém-nascido, sendo de competência dos profissionais de saúde ou parteiras tradicionais responsáveis pela assistência ao parto ou ao recémnascido (reconhecidas e vinculadas às Unidades de Saúde), no caso dos partos hospitalares ou domiciliares ${ }^{8-9}$.

O reconhecimento da importância do monitoramento das informações vitais e a facilidade de acesso aos dados têm resultado no aumento substancial na cobertura e na qualidade das informações de ambos os sistemas. O conhecimento de informações relacionadas às características da mãe e do recém-nascido fornece informações epidemiológicas e serve como subsídio para a avaliação e monitoramento das políticas de saúde materno-infantil, contudo, ainda persiste a precariedade de dados em municípios das regiões menos desenvolvidas ${ }^{6-10}$.

No município de Caiapônia, Goiás, Brasil ainda não existem investigações semelhantes, a realização do estudo irá possibilitar o compartilhamento de experiências com o SINASC em regiões cujas características diferem daquelas já estudas. Desta forma, a realização do presente estudo tem como objetivo caracterizar partos e nascidos vivos de mães residentes no município de Caiapônia, Goiás, Brasil.

\section{Método}

Trata-se de estudo de corte transversal, de abordagem quantitativa, realizado no município de Caiapônia, situado no estado de Goiás, com dados extraídos do SINASC, gerados a partir de $1^{\circ}$ de janeiro a 31 de dezembro de 2016.

O município de Caiapônia localiza-se na região sudoeste do estado de Goiás, faz parte da regional de Saúde Sudoeste II, com população em 2010 de 16.757 habitantes, e população estimada para 2017 de 18.505. Ocupa uma área total de $8.635,129 \mathrm{~km}^{2}$, sendo o $3^{\circ}$ maior de Goiás ${ }^{11}$. Conta com um hospital de médio porte e seis equipes de Estratégia Saúde da Família, distribuídas em cinco Unidades Básicas de Saúde, com cobertura total de $47 \%$ da população.

A Secretaria Municipal de Saúde coordena as ações referentes à Atenção Básica e Núcleo de
Vigilância Epidemiológica, juntamente com todos os Sistemas de Informação referentes a esses setores, incluindo o SINASC. Ao Núcleo de Vigilância Epidemiológica cabem as operações necessárias ao funcionamento desse sistema, que vão desde a distribuição das Declaração de Nascido Vivo nos estabelecimentos de saúde, avaliação sobre o correto preenchimento, até o processamento e análise das informações produzidas, obedecendo às diretrizes propostas pelo Ministério da Saúde ${ }^{8}$.

Quando o parto é hospitalar, a Declaração de Nascido Vivo é preenchida pelo médico ou enfermeiro(a) e obedece ao seguinte fluxo: a primeira via é encaminhada ao Núcleo de Vigilância Epidemiológica para ser digitada no SINASC após revisão feita pela coordenação do setor; a segunda via é entregue à mãe para ser apresentada no cartório no momento do registro civil da criança; e a terceira via fica arquivada no prontuário do neonato no local do nascimento. Caso o nascimento ocorra no domicílio, os pais, acompanhados de testemunhas, devem procurar a Secretaria Municipal de Saúde, onde um profissional do Núcleo de Vigilância Epidemiológica preenche a Declaração de Nascido Vivo, e segue o seguinte fluxo: a primeira via fica no Núcleo de Vigilância Epidemiológica para ser digitada; a segunda e a terceira vias são entregues à mãe para serem apresentadas no cartório para registro da criança e a outra para ser entregue na unidade de saúde onde a criança receberá os primeiros atendimentos.

A amostra foi composta pelos nascidos vivos, filhos de mães residentes no município de Caiapônia, Goiás, Brasil, ocorridos no período de $1^{\circ}$ de janeiro a 31 de dezembro de 2016. A coleta de dados foi realizada no site DATASUS, do Ministério da Saúde, www.datasus.gov.br, seguindo os tópicos: Acesso a informação $>>$ Informações de saúde $($ TABNET) $>>$ Estatísticas vitais $>>$ Nascidos vivos $>>$ Goiás. Dentro da aba de dados que se iniciou após essa seleção, foram selecionados: município, nascimento por residência da mãe, 2016, Caiapônia, Goiás, Brasil.

Foram consideradas as seguintes variáveis: local de ocorrência do parto, sexo e peso do recém-nascido, idade, estado civil e escolaridade da mãe, duração da gestação, tipo de parto, mês de ocorrência do parto e número de consultas de pré-natal. 


\section{Resultados}

Este estudo foi realizado, exclusivamente, com dados secundários e agregados, de acesso público e em conformidade com a Resolução do Conselho Nacional de Saúde (CNS) no 466, de 12 de dezembro de 2012, atendendo os fundamentos éticos e científicos exigidos. O projeto foi dispensado de apreciação por Comitê de Ética em Pesquisa (CEP), conforme recomenda ${ }^{12}$.
No ano de 2016 foram registrados no SINASC, um total de 186 nascidos vivos ${ }^{13}$, os quais predominaram o nascimento no ambiente hospitalar, com percentual de 99,5\%. O único parto ocorrido fora da unidade hospitalar foi registrado na zona rural. Os meses que tiveram maior taxa de nascimento foram em junho e dezembro de 2016, ambos com $11,8 \%$, seguidos pelo mês de maio, com $10,8 \%$ dos nascimentos, e logo após por fevereiro, setembro e novembro, todos com 9,1\% (Tabela 1).

Tabela 1 - Nascidos vivos segundo local e mês de ocorrência do nascimento. Caiapônia, Goiás, Brasil, 2016

\begin{tabular}{lcc}
\hline VARIÁVEIS & N & \% \\
\hline LOCAL DE OCORRÊNCIA & 185 & 99,5 \\
Hospital & 1 & 0,54 \\
Domicílio & & \\
MÊS DE OCORRÊNCIA & 9 & 4,9 \\
Janeiro & 17 & 9,1 \\
Fevereiro & 9 & 4,8 \\
Março & 15 & 8,1 \\
Abril & 20 & 10,8 \\
Maio & 22 & 11,8 \\
Junho & 13 & 7 \\
Julho & 11 & 5,9 \\
Agosto & 17 & 9,1 \\
Setembro & 14 & 7,5 \\
Outubro & 17 & 9,1 \\
Novembro & 22 & 11,9 \\
Dezembro & & \\
\hline
\end{tabular}

Fonte: Dados da pesquisa, 2016.

Em relação às características dos recém-nascidos, observou-se maior número de crianças do sexo masculino (51,7\%) e com peso entre 3000 a 3999g (64\%). (Tabela 2). 
Tabela 2 - Nascidos vivos segundo sexo e peso ao nascer. Caiapônia, Goiás, Brasil, 2016

\begin{tabular}{lcc}
\hline VARIÁVEIS & N & \% \\
\hline SEXO & 96 & 51,7 \\
Masculino & 90 & 48,3 \\
Feminino & & \\
PESO AO NASCER (gramas) & 12 & 6,5 \\
1500 a 2499 & 38 & 20,4 \\
2500 a 2999 & 119 & 64 \\
3000 a 3999 & 17 & 9,1 \\
4000 ou mais & \\
\hline
\end{tabular}

Fonte: Dados da pesquisa, 2016.

Quanto às características da mãe, na idade materna se destacou a elevada frequência de mães adultas, com idades entre 25 e 29 anos, seguidas pelas idades entre 20 e 24 , as quais representaram $26,9 \%$ e $25,8 \%$, respectivamente. Observou-se predominância de mulheres casadas $(40,4 \%)$, seguidas das mães solteiras $(38,2 \%)$. Em relação à escolaridade materna, o percentual de mães com 8 a 11 anos de estudo foi de 53,8\%, seguidos de 12 anos ou mais $(33,9 \%)$ (Tabela 3$)$.

Tabela 3 - Nascidos vivos segundo características maternas. Caiapônia, Goiás, Brasil, 2016

\begin{tabular}{lcc}
\hline VARIÁVEIS & N & \% \\
\hline IDADE (anos) & 1 & 0,5 \\
10 A 14 & 32 & 17,2 \\
15 a 19 & 48 & 25,8 \\
20 a 24 & 50 & 26,9 \\
25 a 29 & 37 & 20 \\
30 a 34 & 17 & 9,1 \\
35 a 39 & 1 & 0,5 \\
40 a 44 & & \\
ESTADO CIVIL & 75 & 40,4 \\
Casada & 71 & 38,2 \\
Solteira & 33 & 17,8 \\
União consensual & 6 & 3,1 \\
Ignorado & 1 & 0,5 \\
Separada judicialmente & & \\
ESCOLARIDADE (anos de & & 11,3 \\
estudo) & 2 & 53,8 \\
1 a 3 & 21 & 33,9 \\
4 a 7 & 100 & \\
8 a 11 & 63 & \\
12 ou mais & & \\
\hline
\end{tabular}

Fonte: Dados da pesquisa, 2016. 
Quanto às características da gestação e dos partos, predominou-se o parto de bebês a termo (37 a 42 semanas), com 93,6\%. O número de partos vaginais $(14 \%)$ foi consideravelmente menor que os partos cesáreos (86\%) e o de mulheres que realizaram de 7 ou mais consultas de pré-natal foi de $68,8 \%$, seguido de $26,3 \%$ que realizaram de 4 a 6 consultas (Tabela 4 ).

Tabela 4 - Nascidos vivos segundo características gestacionais. Caiapônia, Goiás, Brasil, 2016

\begin{tabular}{lcc}
\hline VARIÁVEIS & N & \% \\
\hline DURAÇÃO DA GESTAÇÃO (semanas) & & \\
28 a 31 & 1 & 0,5 \\
32 a 36 & 11 & 5,9 \\
37 a 41 & 174 & 93,6
\end{tabular}

\section{TIPO DE PARTO}

Cesáreo

Vaginal

\section{CONSULTAS DE PRÉ-NATAL}

\begin{tabular}{lcc} 
Nenhuma & 4 & 2,2 \\
1 a 3 & 5 & 2,7 \\
4 a 6 & 49 & 26,3 \\
7 ou mais & 128 & 68,8 \\
\hline
\end{tabular}

86

14

Fonte: Dados da pesquisa, 2016.

\section{Discussão}

As recomendações para que se proceda à revisão e consolidação dos Sistemas de Informação, de forma a permitir a avaliação da cobertura e da qualidade das informações, os dados encontramse em nível satisfatório para a construção de indicadores de desenvolvimento e avaliação políticas de saúde, salvo poucas exceções, que ainda apresentam entraves quanto à quantidade $\mathrm{e}$ qualidade dos dados ${ }^{5,14}$.

Os dados analisados não podem garantir que a totalidade de nascimentos registrados corresponda a real ocorrência de nascimentos na região. Para isso, seria necessário comparar o total de registros do SINASC, com o total de nascimentos por meio de dados hospitalares ou de registros em cartórios. Por outro lado, os dados analisados evidenciaram redução no percentual de preenchimento da categoria "ignorado", o que representa importante melhoria na qualidade do preenchimento e da digitação das variáveis da
Declaração de Nascido Vivo, considerada como um dos aspectos fundamentais para o processo de aperfeiçoamento, gerência e controle da qualidade dos dados ${ }^{3}$.

O ambiente hospitalar destacou-se como o principal local de ocorrência do nascimento no município, com dados semelhantes a outros estudos, onde os percentuais para nascimentos hospitalares chegaram a variar de 97 a 99,6\% $\%^{7,10}$. A relação entre o número de meninos e de meninas foi de $3,23 \%$. A predominância do sexo masculino em comparação ao feminino foi demonstrada também em estudos locais e regionais, que analisaram os nascimentos por períodos de tempo ${ }^{7,10}$.

Entre os indicadores de interesse para a atenção à saúde materno-infantil são imprescindíveis as informações contidas na Declaração de Nascido Vivo, que aponta a proporção de nascidos vivos de baixo peso $^{8}$. O baixo peso ao nascer tem sido associado a vários fatores, como a idade maternal, número de consultas de pré-natal, sexo da criança, 
duração da gestação e escolaridade da mãe, encurtamento da duração da gestação, isolado ou em associação com o retardo de crescimento uterino, deficiência da assistência durante a gestação, entre outras condições ligadas à saúde da mulher, que fazem com que a criança nasça com baixo peso (menos de 2.500 gramas) ${ }^{15}$.

O percentual de crianças com baixo peso ao nascer foi inferior a 7\%. Estudo se assemelha com os dados encontrados, com baixas taxas, variando de 3,8 a $4,6 \%{ }^{10}$. O nascimento de crianças com baixo peso está aumentando no país com o decorrer dos anos, de 7,7\% em 2000 para $8,4 \%$ em 2009. O percentual de nascidos com baixo peso variou de $9,4 \%$ nos municípios de maior porte a $7,4 \%$ nos de menor porte, sendo esses percentuais maiores nas regiões Sul e Sudeste, e menor no estado de Goiás ${ }^{15}$.

O percentual de mães adolescentes foi inferior ao de mães adultas, com uma diferença de 64,6. Um estudo realizado em 2006 mostra elevada prevalência de mães adolescentes, com variações de $27 \%$ a $30 \%$, analisados em um período de três anos ${ }^{10}$. O percentual de mães adolescentes com menos de 20 anos foi de $20 \%$, contra os $26,7 \%$ de mães com 30 anos ou mais de idade 6 . Considerando-se o porte do município de residência da mãe, verifica-se que nos municípios de maior porte, a estrutura etária das mães é muito mais envelhecida do que os de menor porte, onde os que possuem menos de 100 mil habitantes as mães mais jovens são muito mais frequentes do que mães com idades um pouco mais avançadas ${ }^{6}$.

Além de apresentar problemas de natureza fisiológica, a gravideznaadolescência é considerada como gravidez de risco, principalmente, por estar relacionada às condições sócio-culturais que interferem diretamente na evolução da gravidez. A maior parte das adolescentes grávidas pertence a estratos sociais menos favorecidos, sem acesso às informações necessárias durante a gestação e sem adequada assistência pré-natal ${ }^{10}$.

Sobre o estado civil, o percentual de mulheres sem companheiro fixo foi inferior ao de mães casadas, encontrados também em estudo realizado em cidade de pequeno porte ${ }^{10}$. Com a introdução da opção "união estável" dentre as opções de situação conjugal, o percentual de mães "solteira" cai, e aumenta a participação de união estável na mesma proporção ${ }^{16}$.
O campo situação conjugal foi a única variável com algum percentual de "ignorado". Um estudo mostra que apenas as variáveis de "estado civil" e "detecção de anomalia congênita” não mantiveram percentual de ignorados abaixo de $1 \%{ }^{7}$. O SINASC recomenda o preenchimento de todos os campos da Declaração de Nascido Vivo, embora não haja bloqueio na entrada de dados caso algum campo seja deixado em branco ou marcado como ignorado e nem recusa ou devolução de Declaração de Nascido pelo cartório ${ }^{7,10}$.

Quanto ao nível de escolaridade da mãe, mais da metade delas possuem de 8 a 11 anos de estudo, seguido pelas mães que possuem 12 ou mais. Mães com nível de escolaridade inferior a oito anos têm maiores chances de terem recém-nascidos com baixo peso, estando entre as 15 variáveis associadas ao baixo peso ao nascer ${ }^{15}$. A escolaridade da mãe passou a ser coletada com perguntas sobre o nível (ciclos do ensino, divididos em sem escolaridade, ensino fundamental I, ensino fundamental II, nível médio, e superior) e série do ciclo, para adequar a Declaração de Nascido Vivo ao padrão do IBGE. Houve aumento da representatividade da categoria 8 a 11 anos, e respectiva queda na representatividade da categoria 12 anos e mais em algumas regiões após a introdução dos novos formulários ${ }^{16}$.

Das variáveis da Declaração de Nascido Vivo, a escolaridade da mãe apresentou um dos menores percentuais com campos marcados como ignorado, sendo que a raça/cor da pele, Apgar no $1^{\circ}$ e $5^{\circ}$ minutos e anomalia detectada apresentaram proporções com preenchimento ignorado ou em branco em torno ou acima de $4 \%$ em $2009^{6}$.

A duração da gestação é um dos mais importantes fatores determinantes do crescimento intrauterino, pois a partir dela é possível identificar a presença de prematuridade e problemas no desenvolvimento fetal ao verificar a duração e o peso ao nascer ${ }^{10}$. A captação da idade gestacional em semanas, como variável contínua, permite maior precisão na coleta do dado, a redefinição de estratos para cada necessidade, além de cálculo de medidas de tendência central (médias, moda e mediana) ${ }^{16}$.

No presente estudo, mais de $93 \%$ dos partos em 2016 foram de bebês a termo. Dados encontrados em todo o Brasil em 2009 mostram 7\% do total 
de nascidos vivos prematuros (202.214 crianças nascidas com menos de 37 semanas de gestação), com maior percentual em municípios de maior porte populacional, atingindo $8,5 \%$. Entre os períodos de 1982 a 2004, dados do SINASC revelam um aumento da tendência do número de nascimentos pré-termos apresentando valores de $4 \%$ e $10 \%{ }^{6}$.

As informações sobre a idade gestacional para preenchimento da Declaração de Nascido Vivo podem oferecer algum erro, pois são aceitas informações fornecidas pelos sistemas de informação, por registros em prontuários e cartão da gestante, e na falta desses, são aceitas as informações fornecidas pelo companheiro ou pelos familiares da puérpera.

Em relação ao tipo de parto, observa-se predominância de partos cesáreos sobre os partos normais. Nas regiões Sudeste, Sul e Centro-Oeste, observam-se elevadas proporções de parto cesáreo, superiores a $50 \%$, em todas as categorias de porte populacional. Nas regiões Norte e Nordeste, são marcadas as diferenças segundo porte das cidades: nas menores, um pouco mais de $30 \%$ dos partos são cesáreos, enquanto que nos municípios maiores, a proporção de partos cesáreos supera os $50 \%{ }^{6}$. Em um município do estado do Piauí, foi observado um crescimento de partos normais no período de 2000 a $2002^{10}$. No período de 2006 a 2010, a variável "tipo de parto" em todas as regiões do Brasil apresentou percentual de completude dos dados no SINASC acima de 99\%, mostrando a qualidade dos dados analisados a partir desse sistema em todo o país?.

A proporção de mulheres que realizaram 7 ou mais consultas de pré-natal é de quase $70 \%$, seguidas pelas gestantes que realizaram de 4 a 6 consultas, concordando com dados gerais do Brasil que mostram que o percentual de mães com nenhuma consulta reduziu de $4,7 \%$ para $1,8 \%$, entre 2000 e $2009^{6}$. Em 2011, houve uma diminuição no número de gestantes que realizaram de 4 a 6 consultas de pré-natal e um aumento do percentual das gestantes que realizaram 7 ou mais consultas ${ }^{16}$.

Esses achados refletem a importância em realizar o pré-natal, visto que, a maioria dos óbitos de menores de 1 ano ocorre no primeiro mês após o nascimento, pois as gestantes não realizam um pré-natal de qualidade e não garantem uma boa assistência na hora do parto e no cuidado imediato com o recém-nascido. Desta forma, o número de consultas pré-natal tem sido considerado como um dos principais indicadores da qualidade da atenção à saúde da mulher e da criança ${ }^{6-8}$.

\section{Conclusão}

Conclui-se que houve melhoria da qualidade das informações do SINASC, pela redução do percentual de preenchimento da categoria "ignorado", podendo ser considerado como poderoso instrumento para o monitoramento e avaliação da realidade local, servindo de ferramenta para o planejamento de ações no âmbito da saúde da mulher e da criança, e consequentemente, qualidade assistencial a esses grupos.

Apesar da alta cobertura do SINASC no município estudado, é necessário que sejam traçadas novas metas para o seu fortalecimento local, a fim de garantir a cobertura de $100 \%$ dos nascidos vivos, além de envolver profissionais da atenção básica na captação de nascidos vivos, desenvolver atividades educativas sobre gestação, parto e puerpério, realizar treinamento com os profissionais sobre o correto preenchimento da Declaração de Nascido Vivo e possíveis mudanças que venham a ocorrer, alimentar o sistema de informação sobre nascidos vivos, promover o feedback dos dados coletados para os profissionais do sistema local de saúde e estimular mais pesquisas desse cunho em diferentes períodos, que possam subsidiar a tomada de decisões.

\section{Referências}

1. García Pérez C, Aguilar PA. Vigilancia epidemiológica en salud. AMC. 2013;17(6):121-28.

2. Instituto Nacional de Estadísticas y Censo. Unidad de Gestión de Estadísticas Sociodemográficas em Base a Registros Administrativos. Registro Estadístico de Nacidos Vivos 2017. Metodología, 2018.

3. Brasil. Secretaria de Vigilância em Saúde. Departamento de Vigilância Epidemiológica. Guia de vigilância epidemiológica. 7. ed. Brasília, DF: Ministério da Saúde; 2009. 
4. Brasil. Sistemas de Informações sobre Mortalidade (SIM) e Nascidos Vivos (Sinasc) para os profissionais do Programa Saúde da Família. 2. ed. Rev. Atual. Brasília, DF: Ministério da Saúde; 2004.

5. Girodo AM, Campos D, Bittencourt SDA, Szwarcwald CL, França EB. Cobertura do Sistema de Informações sobre Nascidos Vivos e potenciais fontes de informação em municípios de pequeno porte em Minas Gerais, Brasil. Rev Bras Saúde Matern Infant. 2015;15(3):317-24.

6. Brasil. Secretaria de Vigilância em Saúde, Departamento de Análise de Situação em Saúde. Saúde Brasil 2010: uma análise da situação de saúde e de evidências selecionadas de impacto de ações de vigilância em saúde. Brasília, DF: Ministério da Saúde; 2007.

7. Oliveira MM, Andrade SSCA, Dimech GS, Oliveira JCG, Malta DC, Rabello Neto DL, et al. Avaliação do Sistema de Informações sobre Nascidos Vivos. Brasil, 2006 a 2010. Epidemiol Serv Saúde. 2015;24(4):629-40.

8. Brasil. Secretaria de Vigilância em Saúde, Departamento de Análise de Situação de Saúde. Manual de Instruções para o preenchimento da Declaração de Nascido Vivo. Brasília, DF: Ministério da Saúde; 2011.

9. Brasil. Portaria $\mathrm{n}^{\circ} 116$, de 11 de fevereiro de 2009 . Regulamenta a coleta de dados, fluxo e periodicidade de envio das informações sobre óbitos e nascidos vivos para os Sistemas de Informações em Saúde sob gestão da Secretaria de Vigilância em Saúde. Brasília, DF: Ministério da Saúde; 2009.

10. Mascarenhas MDM, Rodrigues MTP, Monte NF. Caracterização dos partos e dos nascidos vivos em Piripiri, Piauí, Brasil. Rev Bras Saúde Matern Infant. 2006;6(2):175-81.

11. IBGE - Instituto Brasileiro de Geografia e Estatística. Acesso em 17 de abril de 2018. Disponível em: https:// cidades.ibge.gov.br/

12. Ministério da Saúde (BR). Conselho Nacional de Saúde. Resolução no 466, de 12 de dezembro de 2012. Diretrizes e normas regulamentadoras de pesquisas envolvendo seres humanos. Diário Oficial [da] República Federativa do Brasil. 2013;150(112 Seção 1):59-62.
13. Brasil. Informações de saúde. Acessado em: 26 de abril de 2018. Brasília, DF: Ministério da Saúde; 2018. Disponível em: http://datasus.saude.gov.br/

14. Frias PG, Szwarcwald CL, Lira PIC. Avaliação dos sistemas de informações sobre nascidos vivos e óbitos no Brasil na década de 2000. Cad Saúde Pública. 2014;30(10):2068-80.

15. Pedraza DF. Baixo peso ao nascer no Brasil: revisão sistemática de estudos baseados no sistema de informações sobre nascidos vivos. Rev Atenção à Saúde. 2014;41(12):37-50.

16. Brasil. Coordenação Geral de Informações e Analises Epidemiológicas - CGIAE, Secretaria de Vigilância em Saúde. Consolidação do Sistema de Informações sobre Nascidos Vivos - 2011. Brasília, DF: Ministério da Saúde; 2013.

\section{Como citar este artigo:}

Silva MCM, Tobias GC, Teixeira CC. Caracterização dos partos e nascidos vivos de mães residentes em um município de Goiás, Brasil. Rev. Aten. Saúde. 2019; 17(61): 21-29. 